\title{
Description of some new syntaxa from the Azores archipelago
}

\author{
José Antonio FERNÁNDEZ PRIETO ${ }^{(\mathbf{1})}$, Carlos AGUIAR ${ }^{(2)} \&$ Eduardo DIAS $^{(3)}$ \\ (1) Área de Botánica. Departamento de Biología de Organismos y Sistemass. Universidad de Oviedo. C/ Catedrático Rodrigo Uría, \\ s/n,330071-Oviedo.España; jafp@uniovi.es \\ (2) CIMO-Centro de Investigação de Montanha. Instituto Politécnico de Bragança.5301-855 Bragança.Portugal; cfaguiar@ipb.pt \\ (3) Departamento de Ciências Agrárias. Universidade dos Açores. Campus de Angra, Terra-Cha 9701-851 Angra do Heroísmo. \\ Portugal; edias@angra.uac.pt
}

\begin{abstract}
:
A new clase Tolpido azoricae-Holcetea rigidi (non grazed perennial plant communities of rocky and earthy scarps, slope deposits and natural forest fringes ); two new orders Tolpido azoricae-Holcetalia rigidi and Frangulo azoricae-Lauretalia azoricae (evergreen broad-leaved micro-mesoforest); four new alliances Thelipterido pozoi-Woodwardion redicantis (comophytic plant communities ), Ornithopo pinnati-gaudion coarctatae (pionner oligotrophic therophitic plant communities), Tolpido suculentae-Agrostion congestiflorae (perennial graminoid communities of cliffs and landslide scarps) and Pericallion malvifoliae (perennial herbs communities of semi-shaded of woodlands), and 14 news associations are described and typified for the Azores archipelago in this paper. Five associations and two alliances are lectotyfied or proposed for the name correction respectively.
\end{abstract}

Key words: New sintaxa, Phytosociology, Azores.

\section{Introduction}

The Azores is probably the last European territory to require a high ranking formal syntaxonomy. In fact, the published phytosociological studies about the vegetation of Azores, following the methods and concepts of the Zurich-Montpellier school, and the rules of the International Code of Phytosociological Nomenclature (Weber et al. 2000), are limited to three publications by D. Lüpnitz in the 1970s (Lüpnitz, 1975a, b and Lüpnitz, 1976), and to the recent description of a noteworthy group of new syntaxa, mainly forests and tall shrublands, among them a new vegetation class, the Lauro azoricae-Juniperetea brevifoliae, by Rivas-Martínez et al. (in RivasMartínez et al. 2002a, b).

We recognize in this paper a new vegetation class, two new orders, four new alliances and thirteen new associations. At the same time, we have carried out a conceptual and nomenclatural revision of some less clear published syntaxa. In the description of the new associations, only the type relevé has been transcribed, and all the new syntaxa have been intentionally depicted in a minimal way. In a previous publication (Fernández Prieto et al., 2006) we presented many of the syntaxa here validated. A comprehensive revision of the vegetation of the Azores will be soon published in Global Geobotany.

Syntaxa numbers follow the same authors. All the described syntaxa, excepting the alliance Thelipterido pozzoi-Woodwardion radicantes, are endemic to the Azores Archipelago.

\section{Syntaxa description}

13. SCHEUCHZERIO PALUSTRIS-CARICETEA NIGRAE Tüxen 1937 nom. mut. Rivas-Martínez \& al. 2002

13b. CARICETALIA NIGRAE Koch 1926 em. Br.-B1. 1948 nom. mut. Rivas-Martínez \& al. 2002

13.3. Anagallido tenellae-Juncion bulbosi $\mathrm{Br}$.-Bl. 1967

13.3.3. Eleocharito multicaulis-Caricetum cedercreutzii F. Prieto \& Aguiar ass. nova hoc loco

Meso- and supratemperate minerotrophic peatlands, usually dominated by Carex cedercreutzii and Eleocharis multicaulis.

Typus associatio: S. Jorge: Serra do Topo, Pedra Vermelha. Altitude $775 \mathrm{~m}$, orientation W, slope $20^{\circ}$, surface $3 \mathrm{~m}^{2}$, cover $70 \%$. Characteristic species: 1 Carex cedercreutzii, 2 Eleocharis multicaulis, 2 Anagallis tenella, + Viola palustris subsp. juresii; Companion species: 3 Agrostis azorica, 1 Potentilla erecta, + Leontodon taraxacoides, + Carex pilulifera subsp. azorica, + Huperzia dentata.

17. Crithmo maritimi-Limonietea Br.-B1. in Br.-B1., Roussine \& Nègre 1952 nom. mut. Rivas-Martínez \& al. 2002

\section{7b. CRITHMO-ARMERIETALIA Géhu 1968}

\subsection{Euphorbio azoricae-Festucion petraeae Lüp- nitz 1975}

Communities of the Azores sea cliffs.

Typus alliancia: Festucetum petraeae Lüpnitz 1976.

Corresponding author: José Antonio Fernández Prieto. Área de Botánica. Departamento de Biología de Organismos y Sistemas. Universidad de Oviedo. C/ Catedrático Rodrigo Uría, s/n, 330071-Oviedo. España; E-mail: jafp@uniovi.es ISSN: 2253-6302 (print)/ISSN: 2253-6515 (on line) CEditaefa DOI: $10.5616 /$ ijgr 120007 
Characteristic species: Azorina vidali, Daucus azoricus, Euphorbia azorica, Festuca petraea, Myosotis maritima, Solidago sempervirens subsp. azorica, Spergularia azorica.

17.6.1. Azorinetum vidalii Lüpnitz 1976 nom. mut [= Campanuletum vidalii Lüpnitz 1976]a

17.6.2. Festucetum petraeae Lüpnitz 1976

17.6.3. Festuco petraeae-Juncetum acuti F. Prieto \& Aguiar ass nova hoc loco

Diagnosis: Communities of Juncus acutus and Festuca petraea inhabiting littoral lava bed depressions filled with coarse texture deposits, temporarily submerged by sea water; sometimes also in rocky seashores.

Typus associatio: Pico: Cachorro. Altitude $0 \mathrm{~m}$, orientation -, slope -, surface $20 \mathrm{~m}^{2}$, cover $50 \%$. Characteristic species: 3 Juncus acutus, 1 Festuca petraea, + Crithmum maritimum; Companion species: + Atriplex prostrata, + Cyrtomium falcatum.

17.6.4. Spergularietum azoricae Lüpnitz 1976

26. ANomodonto Viticulosae-Polypodietea CAMBRICI Rivas-Martínez 1975

26.a. ANOMODONTO VITICULOSAE-POLYPODIETALIA CAMBRICI O. Bolòs \& Vives in O. Bolòs 1957

26.4. Thelipterido pozzoi-Woodwardion radicantis $\mathrm{F}$. Prieto \& Aguiar all. nova hoc loco.

Comophytic communities of rocky or earthy slopes dominated by medium to large sized ferns, present on rainy temperate and Mediterranean hyperoceanic territories, in the Madeiran and Atlantic European provinces.

Typus alliancia: Diplazio caudati -Woodwardietum radicantis, designated here.

Characteristic species: Cystopteris diaphana, Diplazium caudatum, Stegnogramma pozoi, Selaginella azorica and Woodwardia radicans.

26.4.2. Diplazio caudati-Woodwardietum radicantis F. Prieto \& Aguiar ass. nova hoc loco.

Diagnosis: Comophytic community dominated by Woodwardia radicans, with a meso- to supratemperate distribution in the Azorean Sector.

Typus associatio: Faial: Caldera of Faial, way down to the caldera. Altitude $580 \mathrm{~m}$, orientation W, slope $90^{\circ}$, surface $4 \mathrm{~m}^{2}$, cover $80 \%$. Characteristic species: 4 Woodwardia radicans, 2 Selaginella azori$c a,+$ Stegnogramma pozoi, + Diplazium caudatum; Companion species: 1 Athyrium filix-femina, + Blechnum spicant, + Carex guthnickiana, + Osmunda regalis, 1 Sibthorpia europaea.

26.4.3. Selaginello azoricae-Stegnogrammetum pozoi F. Prieto \& Aguiar ass. nova hoc loco

Diagnosis: Thermo- and mesotemperate comophytic community of rocky or earthy slopes, dominated by Stegnogramma pozoi and Selaginella azorica, frequently establishing vegetation mosaic with the $D i$ plazio-Woodwardietum radicantis, although favouring more shaded and disturbed habitats.

Typus associatio: Faial: Fajã Grande, earthy slopes nearby a rivulet. Altitude $c a .100 \mathrm{~m}$, orientation $\mathrm{N}$, slope $90^{\circ}$, surface $1 \mathrm{~m}^{2}$, cover $50 \%$. Characteristic species: 3 Stegnogramma pozoi, 1 Selaginella azorica, + Diplazium caudatum, 2 Cystopteris diaphana.

40. TUberarietea GuttataE (Br.-B1. in Br.-B1., Roussine \& Nègre 1952) Rivas Goday \& Rivas-Martínez 1963 em. Rivas-Martínez 1978 nom. mut. RivasMartínez \& al. 2002

40a. Tuberarietalia guttati Br.-Bl. in Br.-Bl., Molinier \& Wagner 1940 em. Rivas-Martínez 1978 Rivas-Martínez \& al. 2002

40.6. Ornithopo pinnati-Gaudinion coarctatae F. Prieto \& Aguiar all. nova hoc loco

Pioneer, oligotrophic, spring and early summer ephemeral plant communities from the thermomediterranean and thermotemperate vegetation belts of the Azorean Islands. The annual oligotrophic grasslands of Azores are mainly constituted by introduced species, although there is a consistent group of endemic species of Azores, or of Azores and other Macaronesian archipelagos. These two arguments justify the proposal of this new alliance. The lack of basophilous therophyts explains the assignment of the OrnithopoGaudinion coarctatae to Tuberarietalia guttatae.

Typus alliancia: Ornithopo pinnati-Gaudinietum coarctatae, designated here.

Characteristic species: Aichrysum villosum, Gaudinia coarctata, Lolium aristatum and Rostraria azorica.

40.6.1. Ornithopo pinnati-Gaudinietum coarctatae

F. Prieto \& Aguiar ass. nova hoc loco

Diagnosis: Therophytic pioneer phytocoenosis dominated by the Azores endemic annual grass Gaudinia coarctata. It has a strong pioneer character and a manifest preference for recent lava flows ("mistérios").

Typus associatio: Pico: Madalena, Cais do Mourato. Altitude $5 \mathrm{~m}$, orientation -, slope -, surface $3 \mathrm{~m}^{2}$, cover 20\%. Characteristic species: 3 Gaudinia coarctata, 3 Ornithopus pinnatus, 1 Aira caryophyllea, + Lotus hispidus, + Silene gallica, 1 Lotus angustissimus; Companion species: + Leontodon taraxacoides, 1 Plantago coronopus, 1 Polycarpum tetraphyllum.

\section{Tolpido AZORICAE-Holcetea RIGIDI F. Prieto \&}

Aguiar classis nova hoc loco

Non grazed perennial vascular plant communities of rocky and earthy scarps, slope deposits and natural forests fringes, dominated by endemic graminoids, hemicryptophytes and dwarf chamaephytic herbs. It is endemic of the thermomediterranean and thermo- to supratemperate belts of the Azorean biogeographic Province. The majority of the endemic vascular grasses and forbs of the Azores are characteristic of this vegetation class; it is plausible that almost all of the non-endemics found in its communities are allochthonous species. The thorough floristic dissimilarities between the Tolpido-Holcetea rigidi communities and their European and Madeirean counterparts render unattainable their assignation to other already described herbaceous vegetation classes.

Typus classis: Tolpido azoricae-Holcetalia rigidi $\mathrm{F}$ : Prieto \& Aguiar, designated here. 
Characteristic species: Agrostis azorica, Brachypodium gaditanum, Carex guthnickiana, Carex vulcani, Centaurium scilloides, Holcus rigidus, Luzula purpureosplendens, Lysimachia azorica, Scabiosa nitens and Tolpis azorica.

48a. TOLPIDO AZORICAE-HOLCETALIA RIGIDI F. Prieto \& Aguiar ordo novus hoc loco

Sole order of the class.

Typus ordo: Festucion francoi Lüpnitz 1976 corr. F. Prieto, Aguiar, J.C. Costa, Lousã \& Rivas-Martínez corr., designated here.

Characteristic species: See class.

48.1. Festucion francoi Lüpnitz 1976 corr. F. Prieto, Aguiar, J.C. Costa, Lousã \& Rivas-Martínez corr. hoc loco

[=Festucion jubatae Lüpnitz 1976, Beitr. Biol. Pflanzen 51: 223, original name]

Taxonomic correction: Festuca jubata sensu Lüpnitz 1976 et auct. non Lowe should be Festuca francoi Fern.Prieto, C.Aguiar, E.Días \& M.I.Gut. [Bot. J. Linn. Soc. 157(3): 497 (-498; fig. 1), 2008].

Meso-supratemperate, oligotrophic graminoid associations with scattered megaforbs in a matrix of endemic grasses, linked with habitats disturbed by soil and rock mass movements or gusting winds.

Typus alliancia: Potentillo-Agrostietum azoricae Lüpnitz 1975

Characteristic species: Agrostis botelhoi, Agrostis congestiflora subsp. oreophila, Carex punctata, Festuca francoi, Deschampsia foliosa, Euphrasia azorica, Euphrasia grandiflora, Leontodon rigens, Leontodon filii, Myosotis azorica and Veronica dabneyi.

48.1.1. Centaurio scilloides-Deschampsietum foliosae F. Prieto, Aguiar \& Dias ass. nova hoc loco

Diagnosis: Meso- and supratemperate Deschampsia foliosa grasslands subjected to gusting winds.

Typus associatio: Flores: hill in front of Morro Alto. Altitude $850 \mathrm{~m}$, orientation W, slope $20 \%$, surface $4 \mathrm{~m}^{2}$, cover $90 \%$. Characteristic species: 5 Deschampsia foliosa, + Centaurium scilloides, $1 \mathrm{Lu}$ zula purpureo-splendens, + Lysimachia azorica, 1 Agrostis azorica, 2 Festuca francoi; Companion species: + Blechnum spicant, + Erica azorica, + Huperzia dentata, + Juniperus brevifolia, + Carex echinata, + Rubia agostinhoi.

48.1.2. Festucetum francoi Lüpnitz 1975 corr. Fernández-Prieto, Aguiar, J.C. Costa, Lousã \& Rivas-Martínez corr. hoc loco [=Festucetum jubatae Lüpnitz 1975]

Diagnosis: Steep earthy slopes communities of Festuca francoi and Leontodon rigens.

Lectotypus associatio [designated here (Art. 19a of the ICPN)]: table 16, rel. 6 (Lüpnitz 1976: 226). Ilha das Flores: Morro Alto. $230 \mathrm{~m}, 100 \%, 10^{\circ}, 12-$ $15 \mathrm{~m}^{2}$. Assoziations- und Verbandscharakterarten: 4 Festuca francoi (under Festuca jubata), + Leontodon (Picris) rigens. Ordnungs. und Klassencharakterarten: + Potentilla erecta (under Potentilla recta), 1 Calluna vulgaris, 1 Thymus caespitius. Blegleiter: 1 Blechnum spicant, + Lysimachia azorica (under Lysimachia nemorum), + Luzula pur- pureo-splendens, 1 Rubia agostinhoi (under Rubia peregrina), + Carex echinata, + Mentha pulegium, + Plantago coronopus.as1

48.1.3. Lysimachio azoricae-Holcetum rigidi $\mathrm{F}$. Prieto, Aguiar \& Dias ass. nova hoc loco

Diagnosis: Grasslands of slope deposits, frequently with peat formation, present in the meso- and supratemperate belts of the Azorean Sector.

Typus associatio: Flores: Lagoa Branca. Holceta with Sphagnum peat. Primary position. On the edge of a Juniperus brevifolia community. Altitude 200 $\mathrm{m}$, orientation NE, slope $30 \%$, surface $2 \mathrm{~m}^{2}$, cover 80\%. Characteristic species: 4 Holcus rigidus, + Agrostis azorica, + Lysimachia azorica, 1 Potentilla erecta,+ Luzula purpureo-splendens, + Festuca francoi, 2 Deschampsia foliosa; Companion species: + Blechnum spicant, + Lotus pedunculatus, 1 Rubia agostinhoi, + Vaccinium cylindraceum, + Sibthorpia europaea, 2 Sphagnum palustre, 1 Juncus effusus.

48.1.4. Potentillo-Agrostietum azoricae Lüpnitz 1975 [= Potentillo anglicae-Agrostietum azoricae Lüpnitz 1975 corr. Rivas-Martínez, Lousã, F. Prieto, J.C. Costa, Días \& Aguiar 2002]

Diagnosis: Pioneer meso-supratemperate grasslands of Agrostis azorica, particularly common on former ombrogenous blanket bogs removed by mass movements.

Lectotypus associatio [designated here (Art. 19a of the ICPN)]: table 3, rel. 10 (Lüpnitz 1975b: 164). Ilha do Pico: Pico. 800 m. Char.arten: Agrostis azorica (under Agrostis castellana) 3.3; Ordn. und Klass. Char. arten: Potentilla sp. (under Potentilla recta) +.1 , Polygala vulgaris +.1 , Sieglingia decumbens +.1 . Begleiter: Anthoxanthum odoratum 1.1, Blechnum spicant 1.1, Lysimachia nemorum +.1 , Anagallis tenella +.1 , Selaginella azorica (under Selaginella kraussiana) 2.2, Hydrocotyle vulgaris +.1 , Eleocharis multicaulis +.1 , Festuca francoi (under Festuca jubata) 1.1.

48.1.5. Tolpidetum azoricae Lüpnitz 1976

[=Tolpidetum azoricae typische Subassociation, Lüpnitz 1976, Beitr. Biol. Pflanzen 51: 227; excl. Subassociation von Lactuca watsoniana Lüpnitz 1976, Beitr. Biol. Pflanzen, 51: 228]

Diagnosis: Steep earthy slopes communities of Festuca francoi and Leontodon filii.as

Lectotypus associatio [designated here (Art. 19a of the ICPN)]: table 17, rel. 2 (Lüpnitz 1976: 230). Faial: Rand der Caldeira. $850 \mathrm{~m}, 100 \%$, Inklination in Grad $40^{\circ}, 20-25 \mathrm{~m}^{2}$. Charakterarten: 2 Tolpis azorica, + Pericallis (Senecio) malvifolius; Verbancharakterarten: 5 Festuca francoi (under Festuca jubata), 3 Holcus rigidus, + Deschampsia foliosa, + Leontodon filii (under Picris rigens). Ordnungs. und Klassencharakterarten: + Calluna vulgaris, + Potentilla erecta, + Pteridium aquilinum. Blegleiter: 1 Blechnum spicant, + Luzula purpureosplendens, + Lysimachia azorica (sub Lysimachia nemorum), 1 Selaginella azorica (under Selaginella kraussiana), + Athyrium filix-femina, 1 Lotus uliginosus, + Holcus lanatus, + Dryopteris aemula, + Agrostis azorica (under Agrosti castellana). 
48.2. Tolpido succulentae-Agrostion congestiflorae Aguiar \& F. Prieto all. nova hoc loco

Thermomediterranean, occasionally thermotemperate, perennial graminoid communities of cliffs and landslide scarps.

Typus alliancia: Festuco petraeae-Agrostietum congestiflorae Aguiar \& F. Prieto, designated here.

Characteristic species: Agrostis congestiflora subsp. congestiflora, Agrostis gracililaxa, Carex hochstetteriana (dif.), Daucus carota subsp. azorica, Euphorbia azorica (dif.), Festuca petraea and Tolpis succulenta.

48.2.1. Festuco petraeae-Agrostietum congestiflorae Aguiar \& F. Prieto ass. nova hoc loco

Diagnosis: Thermomediterranean and thermotemperate open grassland of rock outcrops, dominated by Agrostis congestiflora, Festuca petraea and Holcus rigidus.

Typus associatio: S. Jorge: Fajã dos Bodes. Altitude $20 \mathrm{~m}$, orientation $\mathrm{S}$, slope $100 \%$, surface $2 \mathrm{~m}^{2}$, cover $40 \%$. Characteristic species: 2 Agrostis congestiflora subsp. congestiflora, 1 Festuca petraea, 1 Holcus rigidus, + Brachypodium gaditanum; Companion species: 1 Thymus caespititius.

48.2.2. Holco rigidi-Brachypodietum gaditanae Aguiar \& F. Prieto ass. nova hoc loco

Diagnosis: Thermotemperate or thermomediterranean mesoxerophilous grassland of Brachypodium gaditanum, in less disturbed habitats co-dominated by Holcus rigidus. Its habitats are small platforms in rock outcrops with soil accumulations provided by solifluction and earth flows.

Typus associatio: S. Jorge: on the way to Fajã de S. João. Altitude $310 \mathrm{~m}$, orientation S, slope 100\%, surface $4 \mathrm{~m}^{2}$, cover $60 \%$. Characteristic species: 3 Brachypodium gaditanum, + Agrostis congestiflora subsp. congestiflora, 1 Holcus rigidus, + Daucus carota subsp. azorica; Companion species: + Euphorbia azorica, 1 Clinopodium vulgare, + Carex hochstetteriana, + Plantago lanceolata.

48.3. Pericallion malvifoliae F. Prieto, Dias \& Aguiar all. nova hoc loco

Perennial herbs communities of semi-shaded well drained external fringes, of Azores endemic woodlands. Present in the thermomediterranean, meso- to supratemperate vegetation belts of the Azorean Sector.

Typus alliancia: Festuco petraeae-Pericallietum malvifoliae F. Prieto, Dias \& Aguiar, designated here.

Characteristic species: Ammi seubertianum, Ammi trifoliatum, Angelica lignescens, Chaerophyllum azoricum, Lactuca watsoniana, Pericallis malvifolia and Ranunculus cortusifolius.

48.3.1. Festuco petraeae-Pericallietum malvifoliae F. Prieto, Dias \& Aguiar ass. nova hoc loco.

Diagnosis: Thermomediterraenan and mesotemperate fringes, sometimes expanding away from forest habitats, of Pericallis malvifolia and Festuca petraea.

Typus associatio: S. Miguel: Nordeste, nearby the sanitary landfill; coastal escarpment; in the edge of an Ericeta azoricae. Altitude $120 \mathrm{~m}$, orientation N, slope $90 \%$, surface $4 \mathrm{~m}^{2}$, cover $80 \%$. Characteristic species: 4 Pericallis malvifolia, 2 Festuca petraea, + Ammi seubertianum; Companion species: 1 Cyrthomium falcatum, 1 Myrica faya, + Petroselinum crispum, + Bromus catharticus.

48.3.2. Lactuco watsonianae-Tolpidetum azoricae (Lüpnitz 1976) F. Prieto, Dias \& Aguiar stat. nov hoc loco

[=Tolpidetum azoricae Subassoziation von Lactuca watsoniana Lüpnitz 1976, Beitr. Biol. Pflanzen 51: 228]

Diagnosis: Mesotemperate fringes.

Lectotypus associatio [designated here (Art. 19a of the ICPN)]: table 17, rel. 7 (Lüpnitz 1975: 230). Açores, Pico: feuchte Schlucht am Cebeço da Cruz. $830 \mathrm{~m}, 100 \%$, Exposition SW, Inklination in Grad $80^{\circ}, 20-25 \mathrm{~m}^{2}$. Charakterarten: 2 Tolpis azorica, + Pericallis malvifolia (sub Senecio malvifolius), + Bellis azorica; Verbancharakterarten: 2 Festuca francoi (under Festuca jubata), 3 Holcus rigidus, 1 Sanicula azorica. Ordnungs. und Klassencharakterarten: + Potentilla erecta, + Polygala vulgaris. Blegleiter: + Blechnum spicant, 1 Luzula purpureosplendens, + Lysimachia azorica (under Lysimachia nemorum), 2 Selaginella azorica (sub Selaginella kraussiana), + Erica azorica, 1 Athyrium filix-femina, + Juncus effusus, 1 Woodwardia radicans, + Holcus lanatus, + Dryopteris aemula, + Huperzia selago, + Sibthorpia europaea, + Euphorbia stygiana, r Laurus azorica, r Cardamine caldeirarum, + Hymenophyllum tunbrigense, $\mathrm{r}$ Vaccinium cylindraceum, r Ranunculus cortusifolius. Außerdem mit geringer Stetigkeit: Viburnum trealeasi (under Viburnum tinus), Mentha pulegium, Fragaria vesca.as1

48.3.3. Agrostio azoricae-Angelicetum lignescentis F. Prieto, Dias \& Aguiar ass. nova hoc loco Diagnosis: Mesotemperate community of Angelica lignescens, inhabiting small rivulets generally dripping from Sphagnum bogs.

Typus associatio: Terceira: Caldeira de $\mathrm{St}^{\mathrm{a}}$ Bárbara, portal da Serra, ca. Lagoa Negra. Altitude 900 m, orientation -, slope -, surface $4 \mathrm{~m}^{2}$, cover $90 \%$. Characteristic species: 3 Angelica lignescens, + Lysimachia azorica, 2 Agrostis azorica, 1 Tolpis azorica, 1 Holcus rigidus; Companion species: 1 Selaginella azorica, 1 Cardamine caldeirarum, + Sibthorpia europaea, 2 Dryopteris azorica, + Potentilla erecta.

49. CALluno VULGARIS-UliCETEA MiNoRIS Br.-Bl. \& Tüxen ex Klika \& Hadač 1944

49a. CALLUNO-ULICETALIA MINORIS Quantin ex Tüxen 1937

\subsection{Daboecion azoricae Lüpniz 1975}

Heathlands of Azores.

Typus: Daboecietum azoricae Lüpniz 1975.

Characteristic species: Daboecia azorica, Diphasiastrum maderense, Huperzia dentata, Huperzia suberecta and Palhinhaea cernua.

49.5.1. Daboecietum azoricae Lüpniz 1975 
Diagnosis: Supratemperate heathlands usually dominated by Daboecia azorica.

Lectotypus associatio [designated here (Art. 19a of the ICPN)]: table 4, rel. 6 (Lüpnitz 1975b: 168). Ilha do Pico: Pico. $1950 \mathrm{~m}, 100 \%, \mathrm{SW}, 45^{\circ}, 2 \mathrm{~m}^{2}$ : Char. arten: Thymus caespititius 4.3, Daboecia azorica 2.2. Diff.arten der typ. Subass.: Silene vulgaris +.1 . Verb. char.arten.: Agrostis congestiflora subsp. oreophila (under Agrostis castellana) +.1. Ordn. und Klass.char.arten.: Calluna vulgaris 1.1, Veronica officinalis +.1 . Begleiter: Blechnum spicant +.1 .

49.5.2. Huperzio dentatae-Callunetum vulgaris F. Prieto, Dias \& Aguiar ass. nova hoc loco

Diagnosis: Mesotemperate heathlands of Calluna vulgaris ("rapa").

Typus associatio: Terceira: on the way to Serra de $\mathrm{St}^{\mathrm{a}}$ Bárbara. Slope with a mineral soil. Altitude 895 $\mathrm{m}$, orientation $\mathrm{S}$, slope $90^{\circ}$, surface $12 \mathrm{~m}^{2}$, cover 80\%. Characteristic species: 4 Calluna vulgaris, 1 Huperzia dentata, + Palhinhaea cernua. Companion species: + Blechnum spicant, + Potentilla erecta, 2 Holcus rigidus, 1 Deschampsia foliosa, 2 Juniperus brevifolia, + Hypochaeris radicata, + Luzula purpureo-splendens, + Centaurium scilloides, 1 Tolpis azorica, + Carex pilulifera subsp. azorica.

57. LAURO AZORICAE-JUNIPERETEA BREVIFOLIAE RivasMartínez, Lousã, F. Prieto, J.C. Costa, Dias \& Aguiar 2002

Azorean evergreen broad-leaved micro-mesoforests, acicular-leaved nano-micro forests and seral tall (microphanerophytic) shrublands, with a hyperoceanic thermomediterranean and thermo- to supratemperate humidultrahyperhumid optimum, mostly inhabiting nutrient poor andosols, that in cold and rainy zones are covered by a thick acid horizon of organic matter or by a deep ombrogenous blanket bog.

Typus classis: Ericetalia azoricae Lüpnitz 1975

Characteristic species: Arceuthobium azoricum, Athyrium filix-femina, Bellis azorica, Blechnum spicant, Cerastium vagans, Carex hochstetteriana, Carex vulcani, Culcita macrocarpa, Dryopteris aemula, Dryopteris azorica, Dryopteris crispifolia, Dryopteris filix-mas, Elaphoglossum semicylindricum, Hedera azorica, Juniperus brevifolia, Myrsine retusa, Platanthera micrantha, Smilax divaricata and Teucrium scorodonia.

57a. ERICETALIA AZORICAE Lüpnitz 1975

Edaphophilous acicular-leaved nano-microforests on leptosols or deeper soils with an iron pan (placic horizon), at higher altitudes covered by a ombrogenous blanket bog, and seral tall shrublands.

Typus ordo: Culcito macrocarpae-Juniperion brevifoliae Sjögren ex Lüpnitz 1975.

Characteristic species: Erica azorica, Grammitis marginella subsp. azorica, Hypericum foliosum, Oreopteris limbosperma, Pteridium aquilinum, Rubus hochstetterorum, R. ulmifolius, and Vaccinium cylindraceum.

\subsection{Culcito macrocarpae-Juniperion brevifoliae} Sjögren ex Lüpnitz 1975

Sole alliance of the order.

Typus alliancia: Daphno-Ericetum azoricae Lüpnitz. 57.1a. Culcito macrocarpae-Juniperenion brevifoliae Rivas-Martínez, Lousã, F. Prieto, J.C. Costa, Dias \& Aguiar 2002

Meso- to supratemperate associations.

Typus suballiancia: Daphno-Ericetum azoricae Lüpnitz 1975.

Characteristic species: Euphorbia stygiana, Viburnum trealeasi, Deschampsia foliosa (dif.), Ilex azorica (dif.), Festuca francoi (dif.).

57.1.1. Cerastio vulgare-Juniperetum brevifoliae Lüpnitz 1975 corr. Rivas-Martínez, Lousã, F. Prieto, J.C. Costa, Dias \& Aguiar 2002

57.1.2. Daphno-Ericetum azoricae Lüpnitz 1975

57.1.3. Euphrasio grandiflorae-Viburnetum treleasei Lüpnitz 1975 corr. F. Prieto \& Aguiar corr. hoc loco

[= Euphrasio grandiflorae-Viburnetum tini Lüpnitz 1976]

Taxonomic correction: Viburnum tinus sensu Lüpnitz 1976 non L. should be Viburnum treleasei Gandoger

Diagnosis: Tall shrublands of Viburnum treleasei.

57.1.4. Picrido filii-Euphorbietum stygianae Lüpnitz 1976

57.1b. Pteridio aquilini-Ericenion azoricae RivasMartínez, Lousã, F. Prieto, J.C. Costa, Dias \& Aguiar 2002 corr. F. Prieto, Dias \& Aguiar hoc loco

[Pteridio pubescentis-Ericenion azoricae Rivas-Martínez, Lousã, F. Prieto, J.C. Costa, Dias \& Aguiar 2002, Itinera Geobot. 15(1): 127, original name]

Thermomediterranean and thermotemperate associations, occasionally lower mesotemperate.

Typus suballiancia: Pteridio pubescentis-Ericetum azoricae Rivas-Martínez, Lousã, F. Prieto, J.C. Costa, Dias \& Aguiar 2002

Characteristic species: Corema azorica, Myrtus communis, Myrica faya (dif.), Carex hostetteriana (dif.), Festuca petraea (dif.).

57.1.5. Festuco petraeae-Coremetum azoricae Rivas-Martínez, Lousã, F. Prieto, J.C. Costa, Dias \& Aguiar 2002

57.1.6. Pteridio aquilini-Ericetum azoricae RivasMartínez, Lousã, F. Prieto, J.C. Costa, Dias \& Aguiar 2002 corr. F. Prieto, Dias \& Aguiar hoc loco

[= Pteridio pubescentis-Ericetum azoricae RivasMartínez, Lousã, F. Prieto, J.C. Costa, Días \& Aguiar 2002, Itinera Geobot. 15(1): 127, original name].

Taxonomic correction: according to Thomson (2000) only Pteridium aquilinum (L.) Kuhn var. aquilinum is present in the Azores archipelago.

57.b. FRANGULO AZORICAE-LAURETALIA AZORICAE F. Prieto, Dias \& Aguiar ordo novus hoc loco

Azorean evergreen broad-leaved edaphoxerophilous, mesic or hygrophilous micro- and mesoforests.

Typus ordo: Dryopterido azoricae-Laurion azoricae Rivas-Martínez, Lousã, F. Prieto, J.C. Costa, Dias \& Aguiar, designated here.

Characteristic species: Frangula azorica, Ilex azorica and Laurus azorica. 


\subsection{Dryopterido azoricae-Laurion azoricae Rivas-} Martínez, Lousã, F. Prieto, J.C. Costa, Dias \& Aguiar 2002

Meso-supratemperate hyperhumid to ultrahyperhumid hyperoceanic Azorean mesic or hygrophilous micromesoforests growing on developed andosols.

Typus alliancia: Dryopterido azoricae-Lauretum azoricae Rivas-Martínez, Lousã, F. Prieto, J.C. Costa, Dias \& Aguiar 2002

Characteristic species: Carex vulcani (dif.), Elaphoglossum semicylindricum (dif.), Prunus azorica, Sanicula azorica.

57.2.1. Culcito macrocarpae-Ilicetum azoricae F. Prieto, Dias \& Aguiar ass. nova hoc loco

Diagnosis: Meso- and supratemperate hyperhumid climatophylous evergreen broad-leaved cloud mesoforests of Ilex azorica, growing on andosols without a placic pan.

Typus associatio: Pico: Boca do Inferno, Misterios de Prainha. Altitude $800 \mathrm{~m}$, orientation -, slope -, surface $200 \mathrm{~m}^{2}$, cover $90 \%$. Characteristic species: 4 Ilex azorica, 2 Juniperus brevifolia, 2 Myrsine retusa, 1 Hypericum foliosum, 2 Vaccinium cylindraceum, + Frangula azorica, 2 Erica azorica, 1 Hedera azorica, 1 Osmunda regalis, 1 Athyrium filix-femina, 3 Blechnum spicant, 1 Carex vulcani, 2 Culcita macrocarpa, 1 Dryopteris affinis, 1 Dryopteris azorica, + Dryopteris crispifolia, 1 Arceuthobium azoricum; Companion species: + Deschampsia foliosa, + Diplazium caudatum, 1 Cardamine caldeirarum, + Bellis azorica, 1 Fragaria vesca, 1 Hydrocotyle vulgaris, 1 Luzula purpureo-splendens, 3 Lysimachia azorica, + Potentilla azorica, + Prunella vulgaris, 1 Rubia agostinhoi, 2 Sibthorpia europaea, 2 Tolpis azorica, + Veronica officinalis, + Viola juresii.

57.2.2. Dryopterido azoricae-Lauretum azoricae Rivas-Martínez, Lousã, F. Prieto, J.C. Costa, Dias \& Aguiar 2002

57.2.3. Woodwardio radicantis-Prunetum azoricae Rivas-Martínez, Lousã, F. Prieto, J.C. Costa, Dias \& Aguiar 2002

\subsection{Myrico fayae-Pittosporion undulati Lüpnitz} 1976

Thermomediterranean or thermotemperate humid micro-mesoforests, frequently strongly disturbed by human activities and often enriched in synanthropic trees and other perennial exotic plants.

Typus alliancia: Myrico fayae-Pittosporetum undulati Lüpnitz 1975 (Lüpnitz 1976: 271, tb. 24) [Art. 20 del ICPN]

Characteristic species: Carex hochstetteriana (dif.), Myrica faya, Picconia azorica.

57.3.1. Carici hochstetterianae-Picconietum azoricae Rivas-Martínez, Lousã, F. Prieto, J.C. Costa, Dias \& Aguiar 2002

57.3.2. Hedychio gardnerani-Pittosporetum undulati Lüpnitz 1976

[= Myrico fayae-Pittosporetum undulati Lüpnitz 1976]

\section{References}

Lüpnitz D. 1975a. Die vertikale Vegetationsgliederung auf der Insel Pico - Azoren. Cuad. Bot. Canar. 23/24: 14-24.

Lüpnitz D. 1975b. Subalpine und alpine Pflanzengesellschaften auf der Insel Pico (Azoren). Bot. Jahrb. 95(2): 149-173.

Lüpnitz D. 1976. Geobotanische Studien zur natürlichen Vegetation der Azoren unter Berücksichtigung der Chorologie innerhalb Makaronesiens. Beitr. Biol. Pflanzen 51: 149-319.

Fernández Prieto JA, Aguiar C, Dias E. 2006. Catálogo Sintaxonómico da Vegetação Vascular da Ilha Terceira. In Dias E, Fernández Prieto JA, Aguiar C. editores. A Paisagem Vegetal da Ilha Terceira (Açores). ALFA- Associação Lusitana de Fitossociologia, Universidade dos Açores: 51-59.

Rivas-Martínez S, Díaz TE, Fernández-González F, Izco J, Loidi J, Lousã M, Penas A. 2002a. Vascular plant communities of Spain and Portugal. Addenda to the Syntaxonomical Checklist of 2001. Part.1. Itinera Geobot. 15(1): 5-432.

Rivas-Martínez S, Díaz TE, Fernández-González F, Izco J, Loidi J, Lousã M, Penas A. 2002b. Vascular plant communities of Spain and Portugal. Addenda to the Syntaxonomical Checklist of 2001. Part.2. Itinera Geobot. 15(2): 433-922.

Thomson JA. 2000. Morphological and genomic diversity in the genus Pteridium (Dennstaedtiaceae). Annals of Botany 85: 77-99.

Weber HE, Moravec J, Theurillat, J-P. 2000. International Code of Phytosociological Nomenclature. 3rd edition. Journal of Vegetation Science 11: 739-768. 